\title{
Front-end electronics for large arrays of macro-bolometers
}

Paolo Carniti* on behalf of the CUORE and CUPID collaborations INFN \& University of Milano Bicocca

E-mail: paolo.carniti@mib.infn.it

Underground bolometric experiments offer unique opportunities for the study of neutrino-less double beta decay and other rare decays. Both the current and next generation detectors consist of large arrays of macro-bolometers. In particular, CUORE is the first bolometric experiment reaching the 1-ton scale and has successfully completed the first physics run. CUPID, CUORE upgrade with particle identification, has also started its first demonstrator (CUPID-0), with very promising performance. The readout of such large scale and high sensitivity bolometric detectors poses new challenges for the front-end and detector calibration electronic systems. We will present the strategies, design choices and actual performance of the electronic instrumentation as implemented in CUORE and CUPID-0.

XVII International Workshop on Neutrino Telescopes

13-17 March 2017

Venezia, Italy

${ }^{*}$ Speaker. 


\section{Introduction}

The discovery of neutrinoless double beta decay $(0 v \beta \beta)$ has been pursued for several decades since it would give an evidence of new physics beyond the Standard Model. $0 v \beta \beta$ is a ultra-rare $\left(T_{1 / 2}^{0 v}>10^{25}-10^{26} y\right)$ process that violates lepton number conservation, whose observation would prove that neutrinos are their own antiparticles (Mayorana particles), and it would also provide information about neutrino mass hierarchy and absolute mass scale.

The bolometric technique has been successfully pioneered in this field of research by other experiments, like Cuoricino and CUORE-0, in the past years and this type of detectors are now among the leading technologies for the search of $0 v \beta \beta$.

CUORE experiment [1] is the first bolometric experiment reaching a 1-ton scale. It uses an array of 988 macro-bolometers made with $\mathrm{TeO}_{2}$, weighting $750 \mathrm{~g}$ each. The experiment is installed underground at Laboratori Nazionali del Gran Sasso (LNGS), Italy. CUORE has successfully completed the first physics run, and is now performing the detector optimization, before the definitive data taking period that will start in autumn 2017.

The sensitivity to $0 v \beta \beta$ is proportional to $\sqrt{\frac{M T}{B \Delta}}$, where $M$ is the detector mass, $T$ is the total data taking time, $B$ is the background rate per units of mass and energy, and $\Delta$ is the energy resolution. In order to maximize the sensitivity, it is beneficial to use high-abundance or enriched isotopes, minimize noise and improve crystal quality for higher energy resolution, and minimize background with contamination reduction or using background rejection techniques.

This last solution is what is pursued in next generation $0 v \beta \beta$ experiments, which aim to reach the zero-background regime, where sensitivity becomes solely proportional on detector mass and experiment life-time. The detection of scintillation or Cherenkov light produced by nuclear events is the most promising technique for particle identification, and thus background rejection, in bolometric experiments.

CUPID [2] experiment will upgrade CUORE by adding particle identification. Its first demonstrator, CUPID-0, adopts enriched scintillating $\mathrm{Zn}^{82} \mathrm{Se}$ crystals read out with germanium light detectors. CUPID-0 has already started data taking with very promising performance.

In this paper, we will present the strategies, design choices and actual performance of the electronic instrumentation for the readout of such large-scale experiments.

\section{Bolometer read out and stabilization}

Bolometers detect any energy deposition in the absorber by producing a small temperature rise, typically about $100 \mu \mathrm{K} / \mathrm{MeV}$ for crystals of a few hundred grams. In order to read out this thermal signal, a temperature sensor is glued to the absorbers. CUORE and CUPID-0 adopt germanium neutron transmutation doped (Ge-NTD) thermistors for this purpose.

When biased with a constant and stable current, the change of resistance due to the temperature rise is converted to a voltage signal as low as $1 \mathrm{mV}$ for a typical $2.6 \mathrm{MeV}$ event. Proper electrothermal feedback is granted by the negative temperature dependence of NTDs. The differential electronic front-end is DC connected to the thermistors and amplifies this small voltage while providing excellent signal-to-noise ratio and high stability over time. 


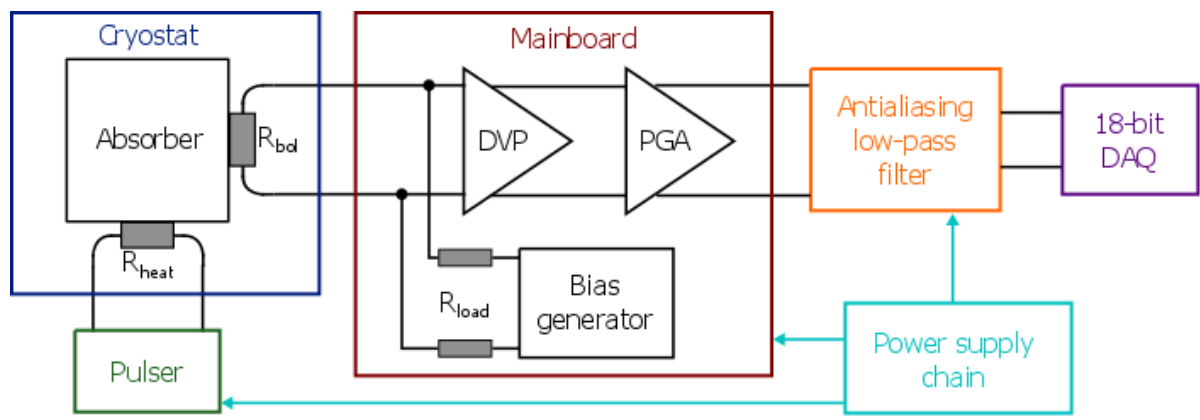

Figure 1: Block scheme of the electronic read out and stabilization of the CUORE detector.

Typical signal bandwidth of macro-bolometers ranges from a few $\mathrm{Hz}$ for bigger crystals, up to a few tens of $\mathrm{Hz}$ for smaller devices like the light detectors of CUPID-0. Due to this characteristics, front-end electronics can be placed at room temperature without the need of a cold amplification stage. NTDs are connected to the front-end with long twisted pairs. In the CUORE cryostat a parasitic capacitance of $500 \mathrm{pF}$ was measured, which gives a bandwidth of $3 \mathrm{~Hz}$ with the typical dynamic detector impedance of $100 \mathrm{M} \Omega$. Common-mode noise and crosstalk on the link are minimized with fully differential readout and accurate cable layout.

The NTD bias current is provided through 2 selectable pairs of load resistors, with total resistances of $10 \mathrm{G} \Omega$ or $60 \mathrm{G} \Omega$, which are polarized with programmable $0 \mathrm{~V}-50 \mathrm{~V}$ bias voltage. Bias current can range from $0.1 \mathrm{pA}$ up to $5 \mathrm{nA}$.

With such high dynamic impedance devices, parallel noise is the main contribution to input noise. NTD intrinsic noise is $0.07 \mathrm{fA} / \sqrt{\mathrm{Hz}}$ at the working temperature of $15 \mathrm{mK}$. The preamplifier input JFETs were specifically selected with a very low gate leakage and their input current noise is about $0.15 \mathrm{fA} / \sqrt{\mathrm{Hz}}$ in differential configuration at a working temperature of $50{ }^{\circ} \mathrm{C}$. The 60 $\mathrm{G} \Omega$ load resistors gives a contribution of $0.55 \mathrm{fA} / \sqrt{\mathrm{Hz}}$, which is the dominant source of parallel noise. Nevertheless, these contributions from the readout electronics are negligible with respect to the best resolutions obtainable with macro-bolometers, which are related with vibrations of the crystals and their thermal dynamics.

For CUPID-0, a larger JFET with lower series noise was selected for an upgraded preamplifier that will be installed in the near future, which will allow the use of NTDs with lower dynamic impedance without increasing the noise contribution due to series noise of the preamplifier.

The complete scheme is shown in Figure 1. The main components and their performance will be described in the following sections.

\subsection{Preamplifier}

The fully differential voltage preamplifier [3, 4], shown in Figure 2, is responsible for the first stage of the voltage signal amplification. Two gain modes are available, high gain at $200 \mathrm{~V} / \mathrm{V}$ and low gain at $20 \mathrm{~V} / \mathrm{V}$. The preamplifier is based on a JFET input pair with selected components to fulfill the low current and series noise requirements in the bandwidth of the signal $(0.1-100 \mathrm{~Hz}$ region). Figure 3 shows typical series noise spectra for CUORE and CUPID-0 preamplifiers.

Output offset, thermal drift and common-mode rejection are remotely settable and are managed by the mainboard hosting the preamplifier. 


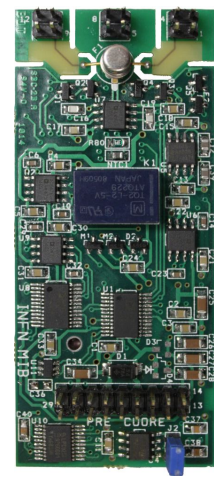

Figure 2: Photograph of the CUORE preamplifier.

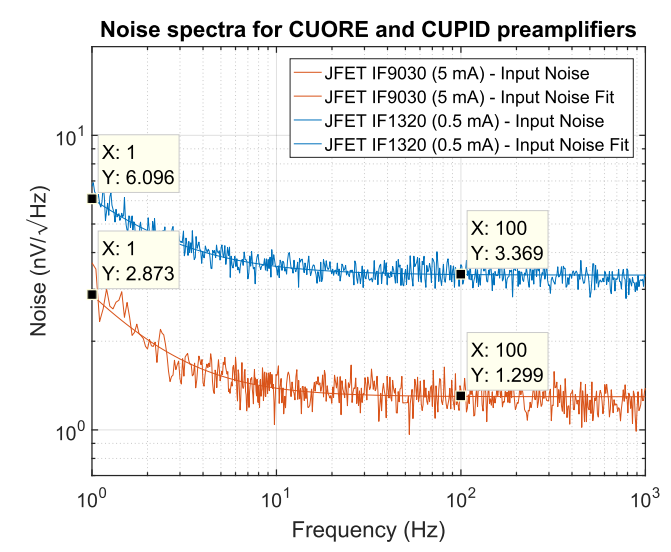

Figure 3: Input series noise of CUORE (blue) and CUPID-0 (red) preamplifiers.

\subsection{Mainboard}

Each mainboard (Figure 4), hosts 6 preamplifiers, handles the second differential stage of amplification with programmable gain (from $1 \mathrm{~V} / \mathrm{V}$ to $50 \mathrm{~V} / \mathrm{V}$ ) and accommodates the detector biasing circuitry. Output offset and detector bias are individually settable for each channel.

The offset is regulated using an intelligent SAR algorithm, with 18-bit resolution. The reference voltages used by the mainboard are provided by a custom linear power supply installed in the mainboard card cage, which will be described later. Thermal drifts and common-mode rejection for each channel were calibrated and optimized during commissioning phase. The residual thermal drift is below $200 \mathrm{nV} /{ }^{\circ} \mathrm{C}$ RMS. The mean CMRR after calibration is greater than $120 \mathrm{~dB}$.

\subsection{Antialiasing filter}

Before being digitized by the 18-bit DAQ, signals are filtered by an active 6-pole BesselThomson filter [5] (Figure 5) in order to suppress high frequency noise and avoid aliasing. The filter has 4 programmable cut-off frequencies that can optimize the bandwidth of each detector. Settable values are 15, 35, 100 and $120 \mathrm{~Hz}$ for CUORE and 15, 100, 140 and $220 \mathrm{~Hz}$ for CUPID-0.

\subsection{Calibration pulser}

The detector energy response is periodically calibrated by injecting voltage pulses through a heater resistance glued on each crystal. These pulses are generated by a low noise, low jitter and high stability pulser board [6] having an amplitude resolution of 12-bit. A photograph is shown in Figure 6. Pulse width jitter is below $1 \mathrm{ppm}$, while pulse amplitude thermal drift is $<0.3 \mathrm{ppm} /{ }^{\circ} \mathrm{C}$. A dedicated pulser board is also responsible of the temperature stabilization of the cryostat mixing chamber with a PC-controlled PID algorithm.

\subsection{Power supply}

Precise, stable and low noise power supplies are crucial for the long and continuous data taking required by bolometric experiments. 


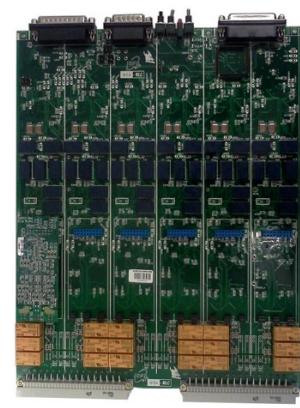

Figure 4: Mainboard populated with a preamplifier.

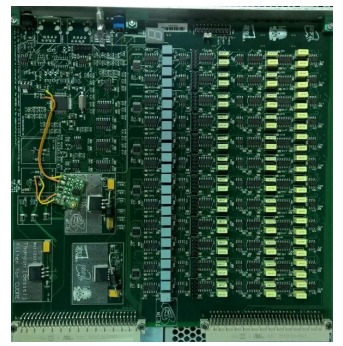

Figure 5: The 12-channel antialiasing filter.

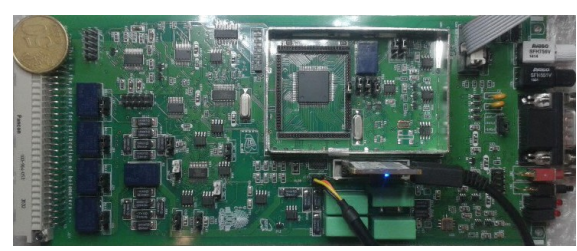

Figure 6: Photograph of the 4-channel stabilization pulser.

The power supply chain was split in three stages. A series of commercial AC/DC converters generate the $48 \mathrm{~V}$ DC voltages fed into the second stage, a custom low noise and insulated DC/DC converter [7]. For those boards where noise and precise references are mandatory (mainboards and pulsers) a third stage, a linear regulator [8], provides more filtered, stabilized and lower noise supplies. These linear regulators have a RMS noise of $240 \mathrm{nV}$ in the $0.1 \mathrm{~Hz}-100 \mathrm{~Hz}$ range, with an absolute precision of $350 \mu \mathrm{V}$ and thermal drifts below $1 \mathrm{ppm} /{ }^{\circ} \mathrm{C}$. Floating supplies allow a star-configured ground connected to the Faraday cage.

\section{Conclusions}

The electronic readout and stabilization systems for CUORE and CUPID-0 detectors are already fully operational and data taking has started for both the experiments. The first physics results are expected in the following months.

\section{References}

[1] D. R. Artusa, et al., Searching for Neutrinoless Double-Beta Decay of ${ }^{130}$ Te with CUORE, Advances in High Energy Physics, 879871 (2015), DOI: https://dx.doi.org/10.1155/2015/879871

[2] D. R. Artusa, et al., First array of enriched $\mathrm{Zn}^{82}$ Se bolometers to search for double beta decay, Eur Phys J, C76 (2016), DOI: https://doi.org/10.1140/epjc/s10052-016-4223-5

[3] C. Arnaboldi, et al., The Design of the Input Stage for the Very Front-End of the CUORE Experiment, J Low Temp Phys, vol.15 (2008), pp. 964-970, DOI: https://dx.doi.org/10.1007/s10909-008-9785-7

[4] C. Arnaboldi, et al., The preamplifier for CUORE, an array of large mass bolometers, IEEE NSS MIC (2009), pp. 389-395, DOI: https://dx.doi.org/10.1109/NSSMIC.2009.5401681

[5] C. Arnaboldi, et al., A programmable multichannel antialiasing filter for the CUORE experiment, Nucl Inst Meth, vol. A617 (2010), pp. 327-328, DOI: https://dx.doi.org/10.1016/j.nima.2009.09.023

[6] P. Carniti, et al., A very high performance stabilization system for macro-calorimeter arrays experiments, IEEE NSS MIC (2016), DOI: https://dx.doi.org/10.1109/NSSMIC.2015.7581791

[7] C. Arnaboldi, et al., Very low noise AC/DC power supply systems for large detector arrays, Review of Scientific Instruments, vol. 86, 124703 (2015), DOI: http://dx.doi.org/10.1063/1.4936269

[8] P. Carniti, et al., A low noise and high precision linear power supply with thermal foldback protection, Review of Scientific Instruments, vol. 87, 054706 (2015), DOI: https://dx.doi.org/10.1063/1.4948390 